\title{
Excessive Dynamic Airway Collapse Detected Using Nondynamic CT
}

\author{
Yukinori Harada and Tomoo Kondo
}

\begin{abstract}
Excessive dynamic airway collapse (EDAC) has been diagnosed using dynamic CT during inspiration and expiration. We herein report an asthma patient with EDAC that was detected incidentally using nondynamic CT. The patient presented with wheezing, cough and mild fever. Treatment for the asthma did not improve her wheeze. CT revealed tracheal narrowing and bulging of the posterior bronchial wall. The patient was diagnosed with EDAC by bronchoscopy. Her wheeze improved with continuous positive airway pressure therapy. Clinicians should be aware of the airway shape when performing nondynamic CT in refractory asthma patients because recognizing the existence of EDAC may help when deciding on the treatment strategy.
\end{abstract}

Key words: excessive dynamic airway collapse, asthma, CT

(Intern Med 55: 1477-1479, 2016)

(DOI: 10.2169/internalmedicine.55.5621)

\section{Introduction}

Excessive dynamic airway collapse (EDAC) refers to an abnormal and exaggerated bulging of the posterior wall within the airway lumen during exhalation. This disease often occurs in patients with asthma or chronic obstructive pulmonary disease (COPD), and many patients do not experience any symptoms or their symptoms are nonspecific (wheeze, dyspnea and cough), which is similar to asthma or COPD (1). EDAC is a relatively new disease entity and it is not well understood by non-pulmonary specialists. It is not so unusual, however, because previous studies reported its prevalence to be $31 \%$ in patients with asthma (2) and $9.4 \%$ in patients with COPD (3). With increasing awareness, the recorded prevalence and detection of EDAC is therefore expected to probably increase in the future.

The gold standard for diagnosing EDAC was the reduction of the airway lumen by $\geq 50 \%$ in terms of the sagittal diameter during forced expiration when observed by bronchoscopy, for which a consensus was reached based on studies that included patients and normal subjects (4). Recently, however, a reduction of cross-sectional airway area by $\geq 50 \%$ during dynamic expiration when observed by $\mathrm{CT}$ has also been used to diagnose EDAC, particularly in elderly pa- tients, because it is noninvasive (1). However, patients must control their breathing during dynamic CT; this is often difficult for elderly patients who are wheezing or dyspneic. Our patient developed EDAC, which was incidentally detected by nondynamic CT. Because the timely recognition of EDAC may help us to understand the severity of asthma and also be helpful in making appropriate treatment decisions, clinicians should therefore be aware of the existence of EDAC in patients with refractory asthma when performing a CT scan.

\section{Case Report}

An 89-year-old Japanese woman developed acute-onset wheezing, dry cough and mild fever. Her symptoms worsened, and she presented to our hospital one day after the onset of symptoms. Her medical history included asthma and Parkinson's syndrome. She did not have any thyroid diseases. She was not a smoker or a drinker. There was no family history of asthma or COPD. She was prescribed levodopa/carbidopa, zonisamide, budesonide inhaler and a tulobuterol patch. She denied any history of trauma, recent changes in medications, accidental ingestions or any other systemic symptoms.

On physical examination, her blood pressure was 116/82 

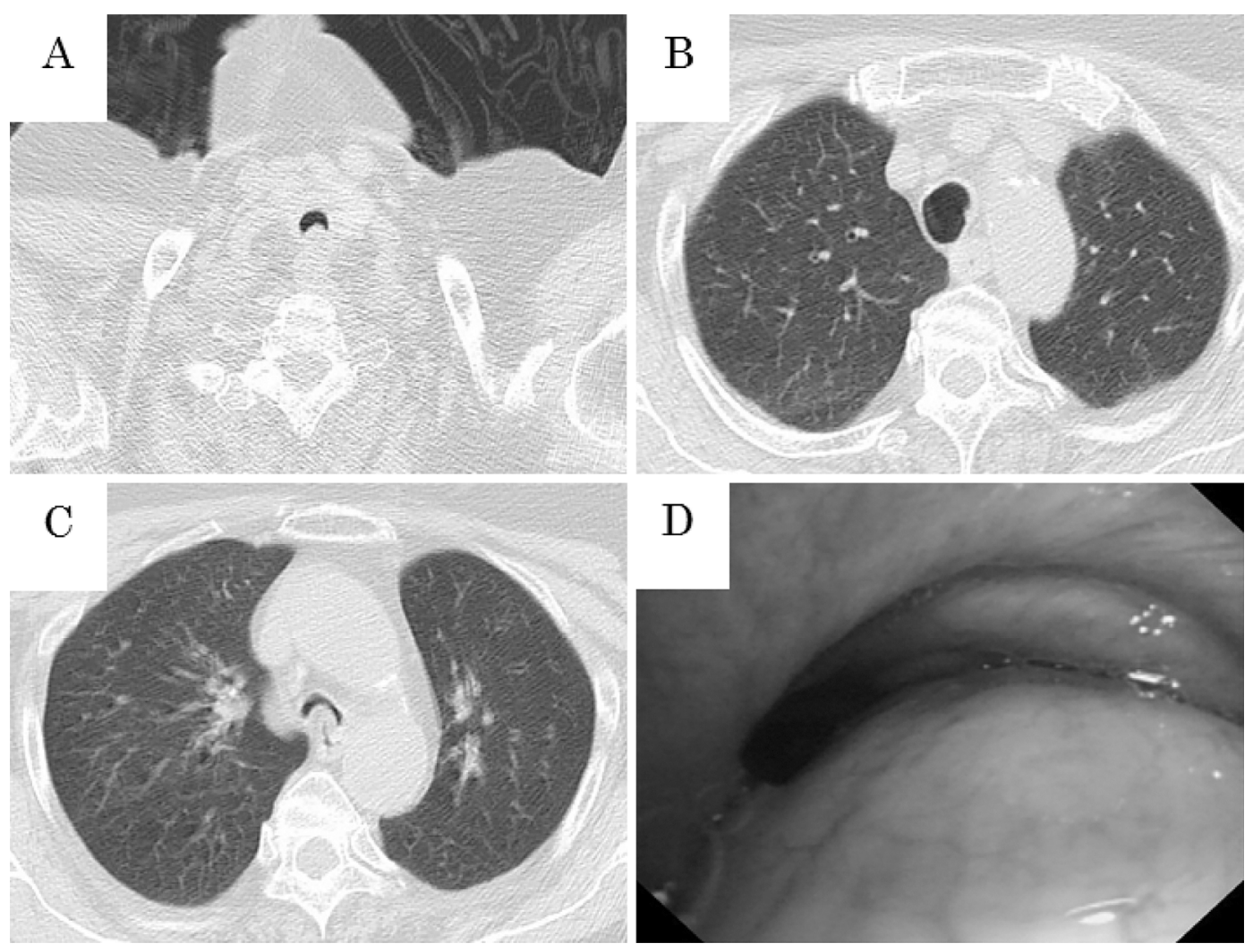

Figure. CT of the chest showing lower tracheal collapse. A CT scan of the upper trachea during expiration (A), middle trachea during inspiration (B) and lower trachea during expiration (C). Bronchoscopy revealing almost complete collapse during expiration (D).

$\mathrm{mmHg}$, pulse 84 beats per minute, respiratory rate 24 breaths per min with an $\mathrm{O}_{2}$ saturation level of $90 \%$ on room air and a body temperature of $36.9^{\circ} \mathrm{C}$. Her neck was supple without jugular venous distension. Cardiovascular examination results were normal. Lung auscultation revealed bilateral, diffuse expiratory wheezing. The findings of an abdominal examination were unremarkable, with no hepatosplenomegaly. Neurological examination revealed muscle rigidity and resting tremor.

A laboratory analysis revealed slightly elevated neutrophils (white cell count $7,700 / \mu \mathrm{L}$, neutrophils $6,100 / \mu \mathrm{L}$, hemoglobin $13.5 \mathrm{~g} / \mathrm{dL}$, platelets $\left.17 \times 10^{4} / \mu \mathrm{L}\right)$, serum creatinine level $(1.02 \mathrm{mg} / \mathrm{dL})$ and C-reactive protein $(0.56 \mathrm{mg} / \mathrm{dL})$. There was no infiltration on her chest X-ray, and the echocardiogram was normal.

The patient was started on an inhaled bronchodilator and systemic methylprednisolone for asthma exacerbation, and her dyspnea, cough and $\mathrm{O}_{2}$ saturation improved. However, her wheeze worsened, and she underwent a CT examination to rule out airway obstruction by a tumor or foreign body. She could not control her breathing and exhaled during the CT examination (Brilliance CT 16, Philips, Cleveland, USA; thin collimation $16 \times 0.75 \mathrm{~mm}$; scan duration 11.56 seconds; scan length $279 \mathrm{~mm}$; reconstruction slice thickness $3.0 \mathrm{~mm}$ ), which incidentally revealed a narrowed airway lumen (Figure A-C). Finally, bronchoscopy revealed an exaggerated bulging of the posterior tracheal wall of and near collapse of the bronchus during exhalation (Figure D). Thus, she was diagnosed with EDAC. Her wheeze improved soon after initiating the treatment, with a continuous positive airway pressure of $6 \mathrm{~cm} \mathrm{H}_{2} \mathrm{O}$ during the night. Three weeks later, her wheeze had disappeared and there was no recurrence.

\section{Discussion}

The specific abnormality of EDAC is exaggerated airway narrowing due to invagination of the posterior membrane during expiration and reduction of the airway lumen by $\geq 50 \%$ in terms of sagittal diameter during forced expiration. This finding is observed using bronchoscopy and is considered the gold standard diagnostic criterion of EDAC. In contrast, a reduction of the airway cross-sectional area by $\geq 50 \%$ between inspiration and expiration observed by dynamic CT has also been recently considered as a diagnostic parameter (4). Another report showed that the timing of the CT is important since the sensitivity of detecting central airway collapse is higher during expiration than during end expiration (5).

The patient in the present report could not control her breathing. A nondynamic CT scan detected EDAC because of its incidental synchronization with one of her breathing cycles at the level of the trachea (Figure A-C). Because we had no data on the two cross-sectional areas during inspiration and expiration at the same level, the patient could not be evaluated on the basis of the CT criterion. However, the abnormal shape of her airway was similar to that seen in pa- 
tients with dynamic expiration reported previously (4), and it was the key factor in recognizing the existence of EDAC.

Our finding was unusual but it was also informative that EDAC can be detected even when using nondynamic CT in tachypneic patients who cannot control their breathing. Our patient's symptoms, other than wheezing, improved after the use of a bronchodilator. However, the effect of bronchodilators on central airways is to produce smooth muscle relaxation, so the tracheobronchial wall stiffness may further decrease after the administration of bronchodilators, and this can worsen the obstruction caused by EDAC. For this reason, Murgu et al. suggested that a comprehensive pretreatment evaluation should be performed to differentiate symptoms attributable to EDAC from those related to underlying pulmonary diseases (4). Thus, although more studies are warranted to clarify the pathology of EDAC, and incidental synchronization with patients' expiration as seen in our case is unusual, it is important to be alert for EDAC when performing a nondynamic CT examination, even when attempting to diagnose other problems.

In conclusion, we should check the shape of the trachea and bronchus in refractory asthmatic and tachypneic patients even when using nondynamic CT because EDAC may be detected by chance.

The authors state that they have no Conflict of Interest (COI).

\section{References}

1. Kalra A, Abouzghelb W, Gajera M, Palaniswamy C, Puri N, Dellinger RP. Excessive dynamic airway collapse for the internist: new nomenclature or different entity. Postgrad Med J 87: 482-486, 2011.

2. Dal Negro RW, Tognella S, Guerriero M, Micheletto C. Prevalence of tracheobronchomalacia and excessive dynamic airway collapse in bronchial asthma of different severity. Multidiscip Respir Med 8: 32, 2013.

3. Represas-Represas C, Leiro-Fernández V, Mallo-Alonso R, Botana-Rial MI, Tilve-Gómez A, Fernández-Villar A. Excessive dynamic airway collapse in a small cohort of chronic obstructive pulmonary disease patients. Ann Thorac Med 10: 118-122, 2015.

4. Murgu SD, Colt HG. Tracheobronchomalacia and excessive dynamic airway collapse. Respirology 11: 388-406, 2006.

5. Baroni RH, Feller-Kopman D, Nishino M, et al. Tracheobronchomalacia: comparison between end-expiratory and dynamic expiratory CT for evaluation of central airway collapse. Radiology 235: 635-641, 2005.

(C) 2016 The Japanese Society of Internal Medicine http://www.naika.or.jp/imonline/index.html 